\title{
An extension of Dragilev's Theorem for the existence of periodic solutions of the Liénard equation
}

\author{
Martina Cioni \\ Dipartimento di Matematica e Informatica "U.Dini", \\ Università, viale Morgagni 67/A, 50137 Firenze, Italy \\ Gabriele Villari* \\ Dipartimento di Matematica e Informatica "U.Dini", \\ Università, viale Morgagni 67/A, 50137 Firenze, Italy
}

\begin{abstract}
The problem of the existence of periodic solution for the Liénard equation is investigated, and a result which improves the classical Dragilev's theorem is presented, together with a corollary in which there are no assumptions on the function $\mathrm{g}(\mathrm{x})$, and hence on $G(x)$, besides the standard sign condition. In the final part of the paper constructive examples with several limit cycles are provided.
\end{abstract}

Keywords: Liénard equation, Limit cycles, Dragilev's Theorem. MSC classification: $34 \mathrm{C} 05,34 \mathrm{C} 25,34 \mathrm{C} 15$.

\section{Preliminaries and well known results}

In this paper we discuss the problem of existence of periodic solutions for the Liénard equation

$$
\ddot{x}+f(x) \dot{x}+g(x)=0
$$

Such a problem has been widely investigated since the first results of Liénard

\footnotetext{
${ }^{*}$ Corresponding author.

Email addresses: martina.cioni@stud.unifi.it (M. Cioni) (Martina Cioni), villari@math.unifi.it (G. Villari) (Gabriele Villari)
} 
[15], appeared in 1928 and there is an enormous quantity of papers on this topic.

It is well known that Liénard equation is equivalent to the system

$$
\left\{\begin{array}{l}
\dot{x}=y \\
\dot{y}=-f(x) y-g(x)
\end{array}\right.
$$

in the phase plane, and to the system

$$
\left\{\begin{array}{l}
\dot{x}=y-F(x) \\
\dot{y}=-g(x)
\end{array}\right.
$$

in the Liénard plane, where $F(x)=\int_{0}^{x} f(x) d x$.

For this reason, the problem of the existence of periodic solutions is bring back to a problem of existence of limit cycles for the previous systems, and in this paper we work in the enviroment of Liénard system (1.2).

Among the existence results until 1960, the classical theorems of Filippov [6], Levinson-Smith [12] and Dragilev [3] may be considered as milestones, while in the last decades the number of results is drammatically increasing.

All the results are based on the classical Poincaré-Bendixson theorem, and in order to fulfil the assumptions of this theorem, it is necessary to produce a winding trajectory large enough.

It is interesting to observe that the methods used to attack this problem are basically two.

We can call the first one the "method of energy", because one may consider the Liénard equation as perturbation of the Duffing equation

$$
\ddot{x}+g(x)=0
$$

which plays the role of the energy.

Let us discuss in details this situation.

\subsection{The method of energy}

The Duffing equation is equivalent in both planes to the system

$$
\left\{\begin{array}{l}
\dot{x}=y \\
\dot{y}=-g(x)
\end{array}\right.
$$


and it is well known that the level curves of the function

$$
H(x, y)=\frac{1}{2} y^{2}+G(x)
$$

where $G(x)=\int_{0}^{x} g(x) d x$, are its solutions.

Here we follow the elegant and concise description of Lefschetz [13, p.266]. If we consider the level curve

$$
\frac{1}{2} y^{2}+G(x)=K
$$

in the dynamical interpretation as motion of a particle, the first term represents its kinetic energy and (1.4) expresses the law of conservation of energy as applied to the particle.

For this reason, we may consider the level curves of the function $H(x, y)$ as energy levels.

Coming back to the Liénard system (1.2), consider a generic point $S=\left(x_{S}, y_{S}\right)$. Keeping the dynamical interpretation, we can say that this point lies on the level of energy

$$
\frac{1}{2} y_{S}^{2}+G\left(x_{S}\right)=K_{S}
$$

For sake of simplicity, we consider a generic point of the y-axis $S=\left(0, y_{S}\right)$, which lies on the level energy $\frac{1}{2} y_{S}^{2}=K_{S}$.

Define as $\gamma^{+}(S)$ the positive semi-trajectory starting from $\mathrm{S}$, and assume that $\gamma^{+}(S)$ moves around the origin and intersects again the y-axis in the same half-plane of $\mathrm{S}$ at a point $R=\left(0, y_{R}\right)$. Clearly, such semi-trajectory is winding if $\left|y_{R}\right|<\left|y_{S}\right|$, unwinding if $\left|y_{R}\right|>\left|y_{S}\right|$ and a cycle if $\left|y_{R}\right|=\left|y_{S}\right|$.

In terms of energy, this means that in the first case we are losing energy, in the second one we are gaining energy and in the last one there is no increment.

Therefore, one can investigate the variation of energy, even if in most papers this was not explicitly observed.

In general, the situation is the following: we start at a point $P=\left(x_{P}, y_{P}\right)$ at the time $t_{0}$ and follow $\gamma^{+}(P)$ for the time $\mathrm{T}$ until the point $Q=\left(x_{Q}, y_{Q}\right)$ at the time $t_{0}+T$.

The variation of energy is

$$
\begin{aligned}
H\left(x_{Q}, y_{Q}\right)-H\left(x_{P}, y_{P}\right) & =\frac{1}{2} y_{Q}^{2}+G\left(x_{Q}\right)-\frac{1}{2} y_{P}^{2}-G\left(x_{P}\right) \\
& =\int_{t_{0}}^{t_{0}+T} \dot{H}(x, y) d t
\end{aligned}
$$




$$
\dot{H}(x, y)=y \dot{y}+g(x) \dot{x}=-y g(x)+g(x)(y-F(x))=-g(x) F(x) \quad
$$

This well known result in the dynamical interpretation shows that when $g(x) F(x)>0$ we are losing energy, while when $g(x) F(x)<0$ we are gaining energy, and in order to have the existence of the limit cycle it is necessary that $g(x) F(x)$ change sign.

Considering the positive semi-trajectory $\gamma^{+}(P)$ reaching the point $\mathrm{Q}$, the variation of energy given by the integral in (1.5) may be split in four parts as follow

$\int_{t_{0}}^{t_{0}+T} \dot{H}(x, y) d t=\int_{\alpha}^{\beta} \frac{-g(x) F(x)}{y-F(x)} d x+\int_{y_{1}}^{y_{2}} F(x) d y+\int_{\beta}^{\alpha} \frac{-g(x) F(x)}{y-F(x)} d x+\int_{y_{3}}^{y_{Q}} F(x) d y$

where $\alpha<0$ and $\beta>0$ are necessary in order to make a correct change of variables.

Such semi-trajectory will be winding if and only if the sum of the four integrals is negative.

We observe that actually the integral

$$
\int F(x) d y
$$

plays a crucial role. This because the integral

$$
\int_{\alpha}^{\beta} \frac{-g(x) F(x)}{y-F(x)} d x
$$

may be considered arbitrarily small.

In principle there are two way for which this can be achivied. The first one is letting the difference $(\beta-\alpha)$ be arbitrarily small. In virtue of the regularity of the function $\frac{-g(x) F(x)}{y-F(x)}$, the integral will be arbitrarily small. On the other hand being the 0 -isocline the y-axis, the trajectory may be read as a function in $\mathrm{y}$, and hence the integral $\int F(x) d y$ is well defined outside the strip $[\alpha, \beta]$. But this way cannot be further developed because in general we cannot evaluate the integral unless we have strong condition on $F(x)$ on the whole line.

The second way is more efficient. The interval $[\alpha, \beta]$ is now fixed and can be large. However, the integral $\int_{\alpha}^{\beta} \frac{-g(x) F(x)}{y-F(x)} d x$ may be considered arbitrarily small if we take a trajectory with $|y|$ arbitrarily large. In this case we can evaluate the integral $\int F(x) d y$, provided that outside the interval $[\alpha, \beta]$, there 
are conditions on $F(x)$ which allow an estimation of the previous integral. For this reason, the role of $\int F(x) d y$ was already emphatized by Lefschetz [[13] p.267], who called this integral taken along a path as the energy "dissipated" by the system.

In order to work with energy, it is therefore necessary to consider trajectories arbitrarily large, and in this light we need sufficient conditions which guarantee that any positive semi-trajectory starting from a point $P=(x, y)$ with $|y|$ arbitrarily large, intersects the vertical isocline $y=F(x)$, otherwise the method fails. Such conditions were at first introduced in [21] and for more general case in [24] (see also [8], [9],[10]).

Most of the existence results present in the literature are actually based on this method, even if, as already mentioned, this was not explicitely stated. One of the most important in this framework is, without any doubt, the classical theorem of Dragilev because the condition of $F(x)$ outside the interval $[\alpha, \beta]$ and the integral $\int F(x) d y$ play a crucial role in the proof.

Theorem 1.1 (Dragilev [3]). Suppose that:

1. $f, g: \Re \rightarrow \Re$ are continuous and $g(x)$ is locally Lipschitz. Also $x g(x)>$ $0 \forall x \neq 0$;

2. $g(x)$ satisfies $x g(x)>0$ for $x \neq 0$ and $\lim _{x \rightarrow \pm \infty} G(x)=+\infty$;

3. $F(x)$ satisfies a Lipschits condition in every finite interval, and $x F(x)<$ 0 for $x \neq 0$ and $|x|$ sufficiently small;

4. There exist constants $N, K_{1}, K_{2}$ with $K_{2}<K_{1}$ such that $F(x) \geq K_{1}$ for $x>N$ and $F(x) \leq K_{2}$ for $x<-N$.

Then the system 1.2 admits at least one limit cycle.

We observe that in order to guarantee that any positive semi-trajectory starting from a point $P=(x, y)$ with $|y|$ arbitrarily large intersects the vertical isocline it is required that $\lim _{x \rightarrow \pm \infty} G(x)=+\infty$.

A similar approach namely the perturbation of a global center may be adopted also when the global center is not given by the Duffing equation. More precisely we split $f(x)$ in $p(x)+h(x)$ and the equation

$$
\ddot{x}+[p(x)+h(x)] \dot{x}+g(x)=0
$$

may be read as a perturbation of the equation 


$$
\ddot{x}+h(x) \dot{x}+g(x)=0
$$

If such equation is a global center, its trajecetories may be considered as the level curves of some kind of energy. But in this case, in general, it is not possible to write the energy explicitely as a function $H(x, y)$ and its variation cannot be express in terms of an integral.

The only possible way seems a comparison of the slopes of the previous two equations and the positive sign of $p(x)$, for $\mathrm{x}$ large, plays a crucial role. Such idea was used in [23], and improved in [24]. More precisely, in [23], the existence of periodic solution was actually proved for the equation

$$
\ddot{x}+\left[x^{3}+x^{2}-1\right] \dot{x}+x^{7}=0
$$

The previous Dragilev's theorem cannot be applied because

$$
\lim _{x \rightarrow \pm \infty} F(x)=+\infty
$$

and hence assumption (4) is not verified.

\subsection{Intersection with the vertical isocline}

The second method to obtain a winding trajectory is strictly related with the intersection with the vertical isocline. More precisely, if there is $\bar{P}=(0, \bar{y})$ with $\bar{y} \neq 0$ such that $\gamma^{-}(\bar{P})$ does not intersect the curve $y=F(x)$ and $\gamma^{+}(\bar{P})$ is oscillatory, clearly such trajectory is winding. Clearly $\gamma^{-}(P)$ is the negative semi-trajectory starting from P.

Such property was called "property K" in [24] where this problem has been investigated.

We observe that, in order to get that $\gamma^{+}(\bar{P})$ is oscillatory, it is again necessary that $\gamma^{+}(\bar{P})$ intersects $y=F(x)$ for $|y|$ large enough.

This method seems more effective because no balance of energy is necessary, but actually, the requirement that $\gamma^{-}(\bar{P})$ does not intersects the vertical isocline is a strong condition on the structure of the system, and in general this implies that $F(x)$ dominates $G(x)$ [24].

An example in which both methods may be used is the Van Der Pol equation

$$
\ddot{x}+\mu\left(x^{2}-1\right) \dot{x}+x=0
$$


and one can easily check that $F(x)$ is $\frac{x^{3}}{3}-x$ while $G(x)$ is $\frac{x^{2}}{2}$ and $F(x)$ actually dominate $G(x)$.

A very recent application in connetion with the uniqueness Massera theorem may be found in [20].

In this paper we use both methods in order to prove the existence of limit cycle for system (1.2) and the main result is a theorem which improves the result of Dragilev, which seems not easy to be further generalized. Moreover we present a corollary in which no assumptions on $g(x)$ are required except for the usual sign condition.

In the final part of the paper, the assumptions of the existence theorem are restricted to an interval $\left[x_{1}, x_{2}\right]$, which depends only on $F(x)$ and $g(x)$.

Such restriction is used to produce constructive examples with several limit cycles.

\section{Main Results}

In the framework of the previous discussion on the role of the energy and the intersection with the vertical isocline, we are going to prove the following result:

Theorem 2.1. Consider the Liénard's equation $\ddot{x}+f(x) \dot{x}+g(x)=0$. Suppose that:

T1: $f, g: \Re \rightarrow \Re$ are continuous and $g(x)$ is locally Lipschitz. Also $x g(x)>$ $0 \forall x \neq 0$;

T2: $g(x) F(x)<0$ for $|x|<\epsilon$ and $x \neq 0$, where $\epsilon$ is arbitrarily small;

T3: Exist $K_{1}, K_{2} \in \Re, K_{1}>K_{2}$ such that

$F(x) \geq K_{1}, x>\beta>0$

$F(x) \leq K_{2}, x<\alpha<0$;

T4: $\lim \sup _{x \rightarrow \pm \infty} G(x) \pm F(x)=+\infty$.

Then, the Liénard's equation has at least one periodic solution.

Proof. We read the Liénard's equation in the equivalent Liénard system (1.2). An usual assumption $T 1$ guarantees existence and uniqueness for its solutions. Moreover the origin is the unique singular point and trajectories are 
clockwise.

Assumption $T 2$ gives that the origin is a source.

In order to prove the existence of at least a stable limit cycle, and hence a non-trivial periodic solution of Liénard's equation, in virtue of the PoncaréBendixson theorem, we need to produce a winding trajectory.

Under assumption $T 3$, assumption $T 4$ is a necessary and sufficient condition for the intersection with the vertical isocline $y=F(x)$ of any positive semitrajectory [21].

The idea of the proof is based on the possibility of producing an arbitrarily large trajectory and, using assumption T3, prove that such trajectory is actually winding.

Assumption $T 3$ gives that $K 1>K 2$, and at first we consider the case $K 1$ positive.

Consider a point $P=\left(\alpha,-y_{P}\right)$ with $y_{P}>0$ arbitrarily large, in virtue of assumption $T 4, \gamma^{+}(P)$ intersects the vertical isocline and, being the slope bounded, intersects again the axis $x=\alpha$ at a point $Q=\left(\alpha, y_{Q}\right)$ with $y_{Q}>0$. Whitout loss of generality, we can assume that $y_{Q} \geq y_{P}$. In fact, if $y_{Q}<y_{P}$, we consider a point $\tilde{Q}=\left(\alpha, y_{P}\right)$ and follow $\gamma^{-}(\tilde{Q})$. There are only two possibilities:

a) $\gamma^{-(\tilde{Q})}$ does not intersect the vertical isocline.

In this case, we already obtain a winding trajectory because, again in virtue of assumption $T 4, \gamma^{+}(\tilde{Q})$ intersects the vertical isocline at first in $\mathrm{x}$ positive and then in $\mathrm{x}$ negative.

b) $\gamma^{-(\tilde{Q})}$ intersects the vertical isocline, and then the $\alpha$-axis at a point $\tilde{P}=\left(\alpha,-y_{\tilde{P}}\right)$ with $y_{\tilde{P}}>y_{P}$, because such negative semi-trajectory is bounded away from $\gamma^{+(P)}$.

Once that this property is taken for granted, we can follow $\gamma^{+}(P)$ until that intersect again the $\alpha$-axis at a point $T=\left(\alpha,-y_{T}\right)$ with $y_{T}>0$.

More precisely, starting at the time $t_{0}, \gamma^{+}(P)$ intersects, as already observe the $\alpha$-axis at the point $Q$, then $\beta$-axis at the point $R=\left(\beta, y_{R}\right)$, then the vertical isocline and again the $\beta$-axis at the point $S=\left(\beta,-y_{S}\right)$ with $y_{S}>0$, and finally the $\alpha$-axis in a point $T$, at the time $t_{1}$.

By contradiction, we assume that such large trajectory is actually unwinding.[Fig.1]

The increment of energy along the trajectory between the point $P$ and the point $T$ is therefore positive. This means that the integral 


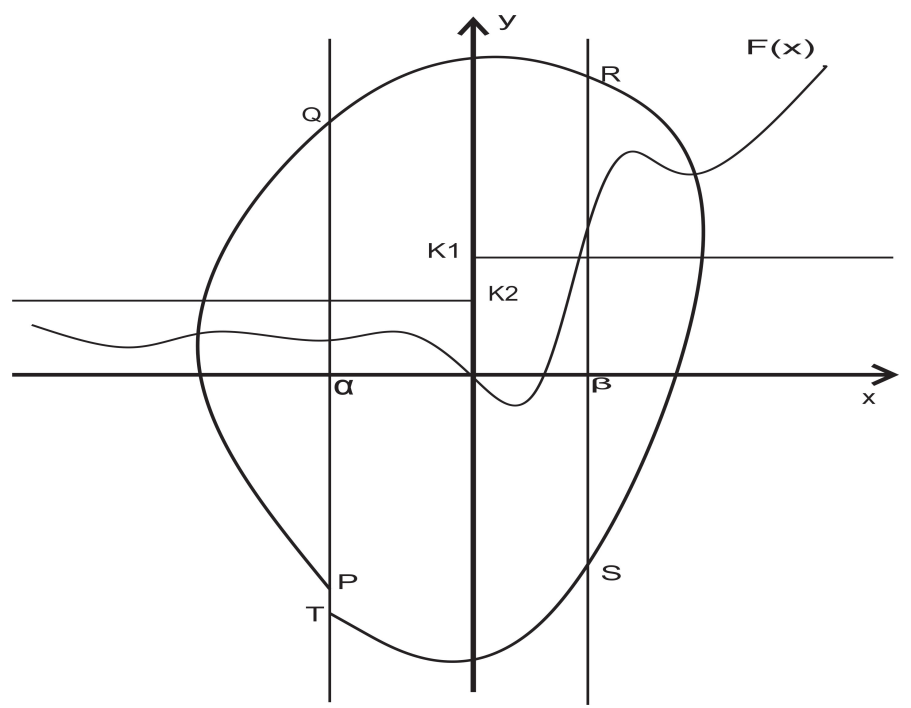

Figure 1: $\gamma^{+}(P)$

$$
\int_{t_{0}}^{t_{1}} \dot{H}(t) d t
$$

taken along the trajectory must be positive.

Let us split such integral in four parts.

$$
\begin{aligned}
\int_{t_{0}}^{t_{1}} \dot{H}(t) d t & =\int_{-y_{P}}^{y_{Q}} F(x) d y+\int_{\alpha}^{\beta} \frac{-g(x) F(x)}{y-F(x)} d x+\int_{y_{R}}^{-y_{S}} F(x) d y+\int_{\beta}^{\alpha} \frac{-g(x) F(x)}{y-F(x)} d x \\
& \leq K_{2}\left(y_{Q}+y_{P}\right)+\int_{\alpha}^{\beta} \frac{-g(x) F(x)}{y-F(x)} d x-K_{1}\left(y_{R}+y_{S}\right)+\int_{\beta}^{\alpha} \frac{-g(x) F(x)}{y-F(x)} d x
\end{aligned}
$$

Observe that $y_{R}$ may be written as

$$
y_{R}=y_{Q}+\Delta_{1} y
$$

where $\Delta_{1} y$ is the unknown increment of $\mathrm{y}$ due to the arc of trajectory in the strip $\alpha \leq x \leq \beta$.

In the same way, $y_{S}$ may be written as

$$
y_{S}=y_{T}-\Delta_{2} y
$$

Clearly, we do not know the sign of $\Delta_{1} y$ and $\Delta_{2} y$. Hence, the previous 
inequality (2.1) may be written as

$$
\begin{aligned}
& K_{2}\left(y_{Q}+y_{P}\right)+\int_{\alpha}^{\beta} \frac{-g(x) F(x)}{y-F(x)} d x-K_{1}\left(y_{Q}+\Delta_{1} y-y_{T}+\Delta_{2} y\right)+\int_{\beta}^{\alpha} \frac{-g(x) F(x)}{y-F(x)} d x= \\
= & K_{2}\left(y_{Q}+y_{P}\right)+\int_{\alpha}^{\beta} \frac{-g(x) F(x)}{y-F(x)} d x-K_{1}\left(y_{Q}+y_{P}\right)-K_{1} \Delta_{1} y-K_{1} \Delta_{2} y+\int_{\beta}^{\alpha} \frac{-g(x) F(x)}{y-F(x)} d x
\end{aligned}
$$

As we can take $y_{P}, y_{Q}$ and $y_{T}$ arbitrarily large

$$
\int_{\alpha}^{\beta} \frac{-g(x) F(x)}{y-F(x)} d x \text { and } \int_{\beta}^{\alpha} \frac{-g(x) F(x)}{y-F(x)} d x
$$

can be considered arbitrary small.

Moreover being the slôpe $\frac{-g(x)}{y-F(x)}$ arbitrarily small in the strip $\alpha \leq x \leq \beta$, the trajectory tends to be orizontal and therefore $\Delta_{1} y$ and $\Delta_{2} y$ can be considered arbitrarily small as well.

Therefore the sign of (2.2) is given by

$$
K_{2}\left(y_{Q}+y_{P}\right)-K_{1}\left(y_{Q}+y_{T}\right)
$$

At this point we must discuss the sign of $K_{1}$ and $K_{2}$ respectively.

We considered the case $K_{1}$ positive. In terms of energy this means that if $x>\beta$ we are "losing" energy. Even if the sign of $K_{2}$ does not play any role, the only interesting case is when $K_{2}$ is positive, because if $K_{2}$ is negative, we are losing energy from both sides and the proof is trivial.

Coming back to (2.3), we assume, by contradiction, that the trajectory is unwinding and hence that actually $\left(y_{Q}+y_{T}\right)>\left(y_{Q}+y_{P}\right)$.

We get

$$
\begin{aligned}
K_{2}\left(y_{Q}+y_{P}\right)-K_{1}\left(y_{Q}+y_{T}\right) & <K_{2}\left(y_{Q}+y_{P}\right)-K_{1}\left(y_{Q}+y_{P}\right) \\
& =\left(K_{2}-K_{1}\right)\left(y_{Q}+y_{P}\right)<0
\end{aligned}
$$

This means that is possible to choose a point $P=\left(\alpha,-y_{P}\right)$ with $y_{P}$ large enough such that $\int_{t_{0}}^{t_{1}} \dot{H}(t) d t<0$ which is the desired contradiction.

On the other hand, if $K_{1}$ is negative, in $x>\beta$ we are "gaining" energy, but being $K_{2}<K_{1}<0$, we are losing energy in $x<\alpha$ and the proof remains the same once that one starts from the point $R$ and construct in a similar way a winding trajectory. 
An evaluation of the amplitude of y will be calculated in the next section in order to give an estimation also in $\mathrm{x}$ and therefore to find a boundary for the limit cycle.

We observe that this result improves the classical theorem of Dragilev [3], where instead of assumption $\mathrm{T} 4$ was required that

$$
\lim _{x \rightarrow \pm \infty} G(x)=+\infty
$$

this because assumptions T3 and (2.4) implies assumption T4.

Moreover, we claim that this result cannot be further improved using the idea of producing a winding trajectory large enough.

In this light, let us analyze the role of all assumptions.

As already mentioned, assumptions T1 and T2 are standard and appear in all the results present in literature.

Assumption T4 cannot be removed or improved.

In fact, an arbitrarily large winding trajectory must clearly intersect the curve $y=F(x)$ for positive time, and such assumption is a necessary and sufficient condition for the intersection with the vertical isocline.

Also assumption T3 is crucial, because otherwise, following the previous discussion concerning the energy, it is possible to produce a large trajectory which is actually unwinding.

As far as we know, the only possible improvement of $T 3$ is the following:

$$
\begin{gathered}
\exists K \in \Re \text { such that } F(x) \geq K \text { for } x>\beta>0 \text { and } \\
\qquad F(x) \leq K \text { for } x<\alpha<0 \\
\limsup _{x \rightarrow+\infty} F(x)>K \\
\liminf \sin _{x \rightarrow-\infty} F(x)<K
\end{gathered}
$$

This assumption may be found in [25, pp.180]. The proof remains the same, because we use the assumptions on $\lim \sup _{x \rightarrow+\infty} F(x)$ and $\liminf _{x \rightarrow-\infty} F(x)$ in order to keep necessary energy inequality. 
The previous theorem has the following corollary:

Corollary 2.1. Assume that:

$C 1: f, g: \Re \rightarrow \Re$ are continuous and $g(x)$ is locally Lipschitz. Also $x g(x)>$ $0 \forall x \neq 0$;

C2: $g(x) F(x)<0$ for $|x|<\epsilon$, with $\epsilon$ is arbitrarily small;

C3: Exists $K \in \Re$ such that

$F(x) \geq K$ for $x>\beta>0$

$F(x) \leq K$ for $x<\alpha<0$;

C4: $\lim \sup _{x \rightarrow+\infty} F(x)=+\infty$ and $\liminf _{x \rightarrow-\infty} F(x)=-\infty$

Then, system (1.2) has at least a stable limit cycle.

Proof. We just observe that assumptions C3 and C4 imply (2.5) and T4 of theorem (2.1).

This result seems interesting because, as far as we know, there is no other result with no assumptions on $g(x)$, besides the regularity and the standard sign condition.

Moreover, we have no sign assumption on $f(x)$ except for the standard one near the origin and no sign assumption on $F(x)$ for $x>\epsilon$ or $x<-\epsilon$.

In this situation let us discuss the rôle of $G(x)$. For sake of semplicity we consider only the case $x>0$.

There are only two possibilities:

1. $\lim _{x \rightarrow+\infty} G(x)<H$

2. $\lim _{x \rightarrow+\infty} G(x)=+\infty$

In the first case we observe that assumption C3 may be relaxed to

$$
F(x) \geq-c \text { for } x>0 \text { and } F(x) \leq c \text { for } x<0, \text { where } c \in \Re^{+}
$$

Because, in this case, the necessary and sufficient condition for intersection with the vertical isocline still holds [21].

Hence, in contrast with $T 3$, we can actually have

$$
F(x) \geq K_{1} \text { whit } x>\beta>0, F(x) \leq K_{2} \text { whit } x<\alpha<0 \text {, where } K_{2}>K_{1}
$$


this because we are not using anymore the idea of a comparison of the energy, but instead the property that there exists a point $P$ such that $\gamma^{-}(P)$ does not intersect $y=F(x)$.

Following the proof of theorem 3 in [21], if we consider a point $P(\bar{x}, \bar{y})$, with $\bar{x}>\beta$ and $\bar{y}<-(\sqrt{2 H}+c)$, we know that $\gamma^{-}(P)$ does not intersect the vertical isocline.

In fact, we consider the function

$$
W(x, y)=\frac{1}{2}(y+c)^{2}+G(x)
$$

The level curves are the phase portrait of the Duffing system shifted by $-c$.

Therefore, if $y<-(\sqrt{2 H}+c)$ and $x>0$, such curves do not intersect the line $y=-c$.

In $x>\beta$, we have

$$
\dot{W}=(y+c) \dot{y}+g(x) \dot{x}=-g(x)[F(x)+c]<0
$$

This means that trajectories of system (1.2) enter such curves and, therefore, back in time, exit from them.

If $\bar{y}<-(\sqrt{2 H}+c)$ and $\bar{x}>0, \gamma^{-}(P)$ does not intersect the line $y=-c$ and, hence, $y=F(x)$.

Clearly $\gamma^{+}(P)$ intersects the vertical isocline in $x<0$ and then in $x>0$, due to assumption $\mathrm{C} 4$, and we get a winding trajectory.[Fig: 2]

We just note that in this particular situation it is possible to obtain the existence of limit cycl with no assumptions on the sign of $F(x)$ and the sign of $f(x)$ as well.

On the other hand, in the second case, assumption $C 3$ or (2.5), cannot be, in general, relaxed.

To investigate such situation, let us go back to the theorem (2.1).

If $F(x)<r$ for $x>0$ large, arguing as before, we can prove that $\gamma^{-}(P)$ intersects the vertical isocline for every $P=(0, y)$, with $y<0$.

More precisely, consider the function

$$
V(x, y)=\frac{1}{2}(y-r)^{2}+G(x)=\text { costant }
$$




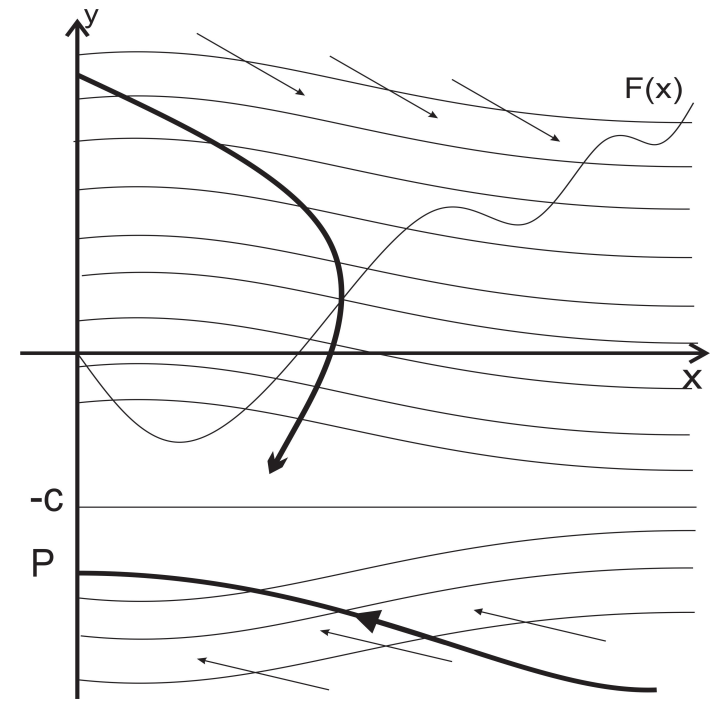

Figure 2: Phase portrait of Duffing system shifted by $-c$ and $\gamma^{-}(P)$

Now, the level curves are the phase portrait of the Duffing system shifted by $+r$, and every curves intersect the line $y=r$.

$$
\dot{V}=(y-r) \dot{y}+g(x) \dot{x}=g(x)[r-F(x)]>0
$$

This means that trajectories of system (1.2) exit such curves and, therefore, back in time, enter in them.

Therefore, $\gamma^{-}(P)$ is guided to line $y=r$ and, hence, intersect the vertical isocline. [Fig: 3]

For this reason, we must apply the idea of the energy, and assumption $C 3$ or (2.5) cannot be relaxed.

Roughly speaking, in order to get that $\gamma^{-}(P)$ does not intersect the vertical isocline, $F(x)$ must 'dominate' $G(x)$.

Otherwise, one has to work with the energy. In this framework relationship between $F(x)$ and $G(x)$ may be found in [24].

Following this work, if $\lim _{x \rightarrow+\infty} G(x)=+\infty$ and $F(x)$ is not bounded above for $x>0$, a necessary and sufficient condition for $\gamma^{-}(P)$ intersecting the vertical isocline is, in our case,

$$
\lim \sup _{x \rightarrow+\infty} \Gamma_{+}(x)-F(x)=+\infty
$$




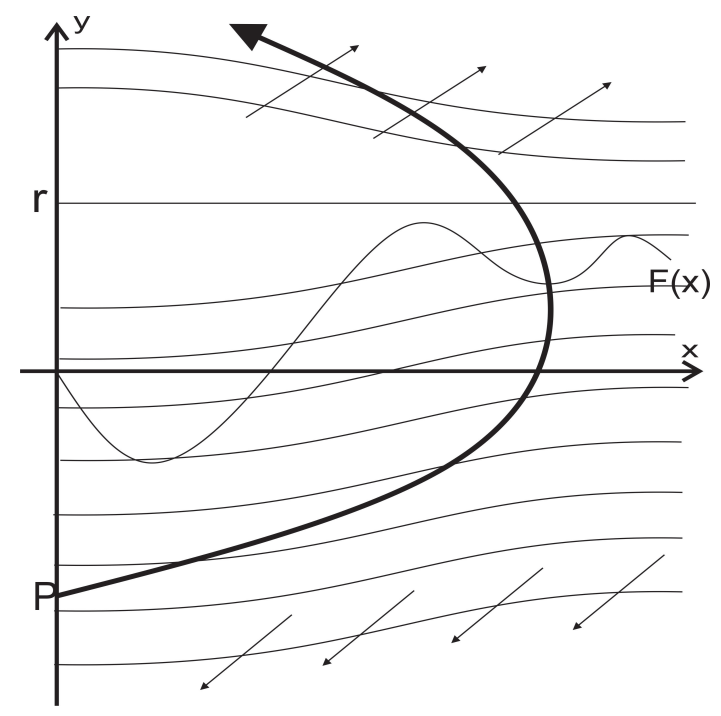

Figure 3: Phase portrait of Duffing system shifted by $+r$ and $\gamma^{-}(P)$

with $\Gamma_{+}(x)=\int_{0}^{x} \frac{g(u)}{1+F_{+}(x)} d u, F_{+}(x)=\max \{0, F(x)\}$, and $\liminf \operatorname{int}_{x \rightarrow+\infty} F(x)<+\infty$.

Therefore, if $\lim \sup _{x \rightarrow+\infty} \Gamma_{+}(x)-F(x)=+\infty$ we need assumption $T 3$, or (2.5).

Otherwise, (2.6) is sufficient.

The case $\lim _{x \rightarrow+\infty} F(x)=+\infty$ is trivial, because one can easily verify that assumption $T 3$ and $T 4$ are verified.

\section{Some estimations on the planar region containing the limit cycle}

In this section, we produce a concrete estimation for the value $y_{P}$ introduced in theorem (2.1).

Such a value will be used in order to get an estimation in $\mathrm{x}$ of the region in which the obtained limit cycle is located.

This problem has already been investigated, for the case $g(x)=x$, in the pioneristic works of J. LaSalle [11] and, for the Van Der Pol equation by Gomory-Richmond [7].

Let us consider a point $A=\left(\beta,-y_{A}\right)$, with $y_{A}>0$. The positive semitrajectory starting from $A$ intersects the y-axis at a point $\bar{B}=\left(0,-\overline{y_{B}}\right)$, with $\overline{y_{B}}>0$.[Fig.4] 


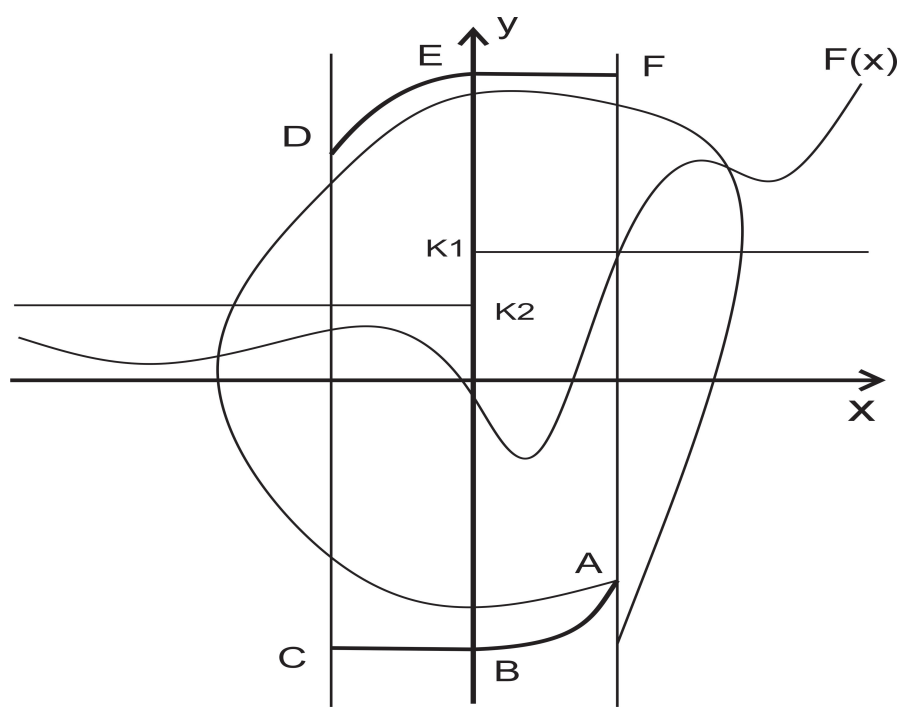

Figure 4: Extimation of $\mathrm{y}$

$$
\begin{aligned}
-\overline{y_{B}} & =-y_{A}+\int_{\beta}^{0} \frac{-g(x)}{y-F(x)} d x=-y_{A}-\int_{0}^{\beta} \frac{-g(x)}{y-F(x)} d x= \\
& =-y_{A}-\int_{0}^{\beta} \frac{g(x)}{F(x)-y} d x \leq-y_{A}-\int_{0}^{\beta} \frac{g(x)}{F_{m}-y_{A}} d x= \\
& =-y_{A}-\frac{1}{F_{m}-y_{A}} \int_{0}^{\beta} g(x) d x=-y_{A}-\frac{G(\beta)}{F_{m}-y_{A}}
\end{aligned}
$$

with $F_{m}=\min _{x \in[0, \beta]} F(x)$.

We call

$$
M_{1}=\frac{G(\beta)}{F_{m}-y_{A}}
$$

and, therefore $\bar{B}$ lies above $B=\left(0,-y_{A}-M_{1}\right)$.

An inspection of the vector field given by system (1.2) shows that $\gamma^{+}(\bar{B})$ intersects the vertical isocline at $\alpha<x<0$ or the $\alpha$-axis at some $-y>-\overline{y_{B}}$. Clearly, we consider only this situation. Consider a point $C=\left(\alpha,-y_{B}\right)$, moving from $\mathrm{B}$ to $\mathrm{C}$ gives an increment of energy of $G(\alpha)$.

This because $H(x, y)=\frac{1}{2} y^{2}+G(x)$ and 


$$
\begin{gathered}
H(B)=\frac{1}{2} y_{B}^{2} \\
H(C)=\frac{1}{2} y_{B}^{2}+G(\alpha)
\end{gathered}
$$

For this reason, from now on, we consider $\gamma^{+}(C)$. Working as in corollary (2.1) and using the function $W_{2}(x, y)=\frac{1}{2}\left(y-K_{2}\right)^{2}+G(x)$, we get

$$
\begin{aligned}
W_{2}(\dot{x}, y) & =-\left(y-K_{2}\right) g(x)+g(x)(y-F(x)) \\
& =K_{2} g(x)-g(x) F(x)=-g(x)\left[F(x)-K_{2}\right]<0
\end{aligned}
$$

Hence $\gamma^{+}(C)$ intersects again the $\alpha$-axis in $y>0$ below the point $D=\left(\alpha, y_{D}\right)$ with $y_{D}=y_{C}+2 K_{2}$.

As before, we consider a point $E=\left(0, y_{E}\right)$ with $y_{E}=y_{D}+M_{2}$, where $M_{2}=\frac{G(\alpha)}{y_{D}-F_{M}}$ with $F_{M}=\max _{x \in[\alpha, 0]} F(x)$, and the point $F=\left(\beta, y_{E}\right)$.

We know that $\gamma^{+}(D)$ intersects the y-axis below $\mathrm{E}$ and $\beta$-axis below $\mathrm{F}$. Summarising this costruction, we can say that $\gamma^{+}(A)$ or intersects the $\mathrm{x}$-axis in $0<x<\beta$, and it is clearly winding, or the $\beta$-axis in y positive below $\mathrm{F}$.

Our goal is to proof that such trajectory is winding, that is to proof that $\gamma^{+}(F)$ itersects the $\beta$-axis at a point $\mathrm{G}$ above $\mathrm{A}$.

We assume, by contradiction, that this is not the case and evaluate the variation of energy, as we did in (2.1).

The increment of energy along the trajectory between the point A and the point $\mathrm{G}$ is given by

$$
\begin{aligned}
\int_{t_{0}}^{t_{1}} \dot{H(t)} d t=H(G)-H(A) \\
=\frac{1}{2}\left(-y_{G}\right)^{2}+G(\beta)-\frac{1}{2}\left(-y_{A}\right)^{2}-G(\beta) \leq \frac{1}{2}\left(y_{F}-2 K_{1}\right)^{2}-\frac{1}{2} y_{A}^{2}= \\
=\frac{1}{2} y_{F}^{2}+2 K_{1}^{2}-2 K_{1} y_{F}-\frac{1}{2} y_{A}^{2}= \\
=\frac{1}{2}\left(y_{A}+M_{1}+M_{2}+2 K_{2}\right)^{2}+2 K_{1}^{2}-2 K_{1}\left(y_{A}+M_{1}+M_{2}+2 K_{2}\right)-\frac{1}{2} y_{A}^{2} \\
=\frac{1}{2}\left(y_{A}^{2}+M_{1}^{2}+M_{2}^{2}+4 K_{2}^{2}+2 y_{A} M_{1}+2 y_{A} M_{2}+4 K_{2} y_{A}+2 M_{1} M_{2}+4 K_{2} M_{1}+4 K_{2} M_{2}\right)+ \\
\quad+2 K_{1}^{2}-2 K_{1} y_{A}-2 K_{1} M_{1}-2 K_{1} M_{2}-4 K_{1} K_{2}-\frac{1}{2} y_{A}^{2}= \\
=\frac{\left(M_{1}+M_{2}\right)^{2}}{2}+2 K_{2}^{2}+y_{A} M_{1}+y_{A} M_{2}+2 K_{2} y_{A}+2 K_{2} M_{1}+2 K_{2} M_{2}+2 K_{1}^{2}- \\
\quad-2 K_{1} y_{A}-2 K_{1} M_{1}-2 K_{1} M_{2}-4 K_{1} K_{2}= \\
=2\left(K_{2}-K_{1}\right) y_{A}+M_{1}\left(y_{A}+2 K_{2}-2 K_{1}\right)+M_{2}\left(y_{A}+2 K_{2}-2 K_{1}\right)+\left(K_{2}-K_{1}\right)^{2}+\frac{\left(M_{1}+M_{2}\right)^{2}}{2}
\end{aligned}
$$

Now, recalling the definition of $M_{1}$ and $M_{2}$, it is clear that the leading term 
of the latter expression is $2\left(K_{2}-K_{1}\right) y_{A}$ and, being $K_{1} \geq K_{2}$, it has negative sign. Therefore, it may be found $\widetilde{y}$ large enough such that the expression is negative. This is actually the desidered value of $\mathrm{y}$, that is any positive semitrajectory starting below $A=\left(\beta,-y_{A}\right)$, with $-y_{A} \leq-\widetilde{y}$ is actually winding. However it is possible to simplify the above expression using the fact that

$$
\lim _{y_{A} \rightarrow+\infty} M_{1}=0, \lim _{y_{A} \rightarrow+\infty} M_{2}=0
$$

and getting a second order degree inequality, which is elementary to solve. We are now able to get the $\mathrm{x}$ estimation. Consider again the point $F=$ $\left(\beta, y_{F}\right)$ and assume that $\lim _{x \rightarrow+\infty} G(x)=+\infty$.

We already used the fact that $\gamma^{+}(F)$ is constrained by the solutions of the shifted Duffing equation, given by $\frac{1}{2}\left(y-K_{1}\right)^{2}+G(x)=H$.

We consider the level of energy identified by the point $F=\left(\beta, y_{F}\right)$, namely $\frac{1}{2}\left(y_{F}-K_{1}\right)^{2}+G(\beta)$, which we denote with $H_{1}$.

The level of energy $H_{1}$ intersects the axis $y=K_{1}$ at a point $\widetilde{x}=G^{-1}\left(H_{1}\right)$ and this is our positive boundary in $\mathrm{x}$.

This argument clearly fails if $G(x)$ is bounded. But in this case we must have $\lim \sup _{x \rightarrow+\infty} F(x)=+\infty$ and, therefore, is sufficient to take in $x>\beta$ any value of $F(x)$ such that $F(x) \geq y_{F}$.

The same argument can be used to find the negative $\mathrm{x}$ boundary.

At this point, we are able to locate the limit cycle.

As a side remark, we notice that such estimation is not the best possible, but it is enough for our purpose. Such a result was expected, and in principle is possible to see it using any scentific computing enviroment, but as far as we know it was never explicitly written.

A first and natural conseguence of the previous estimation is the following. Consider the system (1.2) with $F(x)$ and $g(x)$ fixed and satisfying the assumptions of theorem (2.1). Such assumptions, which are required in the whole line, may be relaxed to the interval $\left[x_{1}, x_{2}\right]$ where $x_{1}$ and $x_{2}$ can be determined.

A natural conseguence is that $F(x)$ and $g(x)$ can be arbitrarily modified outside the interval $\left[x_{1}, x_{2}\right]$ and this can be used in order to produce concrete examples with several limit cycles. Below we present an example with at least two limit cycles.

This problem has been widely investigated in literature. The first result in this direction is due to Levinson-Duff [4], where there is an example in which 
three limit cycles are provided for the equation $\ddot{x}+\epsilon f(x) \dot{x}+x=0$. This result was achivied using the Poincaré method of small parameters [14] and is valid for $\epsilon$ arbitrarily small. We observe that such a method can be actually used only for the case $g(x)=x$. Conversely our result, in virtue of the corollary (2.1), is valid for any $g(x)$ given, provided that $x g(x)>0$.

The same method was used by Lins, De Melo, Pugh [16] in order to state their well known conjectures concerning the upper-number of limit cycles for the polynomial equation $\ddot{x}+p^{n}(x) \dot{x}+x=0$, where $p^{n}(x)$ is an arbitrariypolynomial of degree $\mathrm{n}$ (see also [2] [1] where the weak conjectures was actually proved in the case $\left.\ddot{x}+\epsilon p^{n}(x) \dot{x}+x=0\right)$.

In a very recent paper Dumortier, Panazzolo,Roussarie [5], proved that actually the Lins-DeMelo-Pugh conjectures fails for $n=7$. This very interesting and deep result was proved working with the system $\epsilon \ddot{x}+p^{7}(x) \dot{x}+x=0$, and producing, for $\epsilon$ small, an example with four limit cycles. Finally Riskov [19] proved that equation $\ddot{x}+\left(a x^{4}+b x^{2}+c\right) \dot{x}+x=0$ has at most two limit cycles (for other related results one can see the works of Loyd [17],[18]).

Example: Given the system (1.2) with $F(x)$ and $g(x)$ fixed and satisfying the assumptions of theorem (2.1), one can find the interval $\left(x_{1}, x_{2}\right)$ as before and set

$$
\begin{gathered}
F_{1}(x)=F(x) \text { in }\left(x_{1}, x_{2}\right) \\
\lim _{x \rightarrow+\infty} F_{1}(x)=-\infty \\
\lim _{x \rightarrow-\infty} F_{1}(x)=+\infty
\end{gathered}
$$

Then, the system

$$
\left\{\begin{array}{l}
\dot{x}=y-F_{1}(x) \\
\dot{y}=-g(x)
\end{array}\right.
$$

has at least two limit cycles.

In order to costruct the second limit cycle, we must investigate two situations:

1. Every positive semi-trajectories starting from $P=\left(x_{2}, y_{P}\right)$ with $y_{P}$ arbitrarily large intersect the vertical isocline $y=F_{1}(x)$ and the same holds for a point $Q=\left(x_{1},-y_{Q}\right)$ with $y_{Q} \geq 0$ arbitrarily large. In this case we can argue as in theorem (2.1), but now it is possible to find 
$\overline{K_{1}}<\overline{K_{2}}$ such that $F_{1}(x)<\overline{K_{1}}$ for $x>\bar{\beta}$ and $F_{1}(x)>\overline{K_{2}}$ for $x<\bar{\alpha}$, and we obtain an unwinding trajectory.

2. If one of the assumptions of case (1) fails, we argue as follow: assume that exists a point $\bar{P}=\left(x_{2}, \overline{y_{P}}\right)$ such that $\gamma^{+}(\bar{P})$ does not intersect the vertical isocline. It is easy to see that $\gamma^{-}(\bar{P})$ intersects the vertical isocline for $x<0$ and $y>0$ and hence we get an unwinding trajectory. The same for a point $\bar{Q}=\left(x_{1},-\overline{y_{Q}}\right)$.

In both cases, as usual, the Poincaré-Bendixson theorem gives the second unstable limit cycle.

This procedure can be iterated and in principle, with more tedious calculation, one can construct an example with $n$ limit cycles being $n$ any natural number.

\section{References}

[1] Albrecht, F., Polynomial Liénard equations and Hilbert's 16th problem, Proceeding of international meeting on ordinary differential equations and their applications, Florence (Italy), semptember 20-24, 1993.

[2] Albrecht, F.,Villari,G., Periodic orbits of planar polynomial Liénard systems with a small parameter, Lecture Notes in Math. 1475, Springer, Berlin, 1991, pp 41-52.

[3] Dragilev A.V, Periodic solution of a differential equation of nonlinear oscillation, Prikladnaya mat. i. mek., 16, pp. $85-88$ (Russian).

[4] Duff, G.F.D., Levinson, N., On the non-uniqueness of periodic solutions for an asymmetric Liénard equation, Quart. Appl. Math. 10 (1952) 86-88.

[5] Dumortier, F., Panazzolo, D.,Roussarie, R., More limit cycles than expected in Liénard systems, Proc. Amer. Math. Soc. 135, (2007) 1895-1904.

[6] Filippov, A.F.,A suffcient condition for the existence of a stable limit cycle for a second order equation, (Russian), Mat. Sb. 30 (1952), 171180.

[7] Gomory, R., Richmond, D.E., Boundaries for the limit cycle of Van der Pol's equation, Quart. Appl. Math, 9(1951), 205-209. 
[8] Graef, J.R., On the generalized Liénard equation with negative damping, J. Differential Equations, 12 (1972), 393-402.

[9] Hara, T., Sugie, J., When all the trajectories in the Liénard plane cross the vertical isocline?, NoDEA 2 (1995) 527551.

[10] Hara, T., Yoneyama, T., On the global center of generalized Liénard equation and its application to stability problems, Funkc. Ekvac. 28 (1985) 171192 .

[11] LaSalle, J., Relaxation oscillations, quart. Appl. Math, 7 (1949), 1-19.

[12] Levinson, N., Smith, O.K., A general equation for relaxation oscillations, Duke Math. J., 9 (1942), 382-403.

[13] Lefschetz,S., Differential Equations: Geometric Theory, Interscience Publishers, Inc., New York, 1957.

[14] Lefschetz, S., Lectures on Differential Equations, Princeton University Press, (1948), 194-204.

[15] Liénard, A., Étude des oscillations entretenues, Revue génér. de l'électr. 23, (1928), 901-902; 906-954.

[16] Lins, A., De Melo, W., Pugh, C.C., On Liénard's equation, Lecture notes in Mathematics 597, Springer, New York 1976, 335-357.

[17] Lloyd, N.G., Liénard systems with several limit cycles, Math. Proc. Cambridge Phi- los. Soc. 102 (1987), 565-572.

[18] Lloyd, N.G., Lynch, S. Small amplitude limit cycles of certain Liénard systems, Proc. Roy. Soc. London Ser. A 418 (1988), 199-208.

[19] Riskov, G. S., The maximal number of limit cycles of the system $\dot{y}=-x$, $\dot{x}=y-\sum_{2}^{i=0} a_{i} x^{2 i+1}$ is equal to two, Differencial $\eta \gamma e$ Uravnenija, 11 (1975), 390-391, 400. (Russian)

[20] Rosati, L., Villari, G., On Massera's Theorem concerning the uniqueness of a periodic solution for the Liénard equation. When does such periodic solution actually exist?, Boudary Values Problems 2013, (2013:144). 
[21] Villari, G., On the qualitative behaviour of solutions of Liénard equation, Journal of differential equations 67.2 (1987).

[22] Villari, G. Periodic Solutions of Liénard's Equation, J. Math. Analysis Appl. 86 (1982).

[23] Villari, G., On the existence of periodic solutions for Liénard's equation, Nonlinear Analysis, TMA 7 (1983), 71-78.

[24] Villari, G., Zanolin, F., On a dynamical system in the Liénard Plane. Necessary and Sufficient Conditions for the Intersection with the Vertical Isocline and Applications, Funkcial. Ekvac. 33 (1990).

[25] Zhang Zhi Fen et al; Qualitative Theory of Differential Equations , Traslations of Mathematical Monographs, AMS volume 101, (1991). 